\title{
Biochemical characterization of trans-sialidase TS1 variants from Trypanosoma congolense
}

\author{
Hendrik Koliwer-Brandl, Thaddeus T Gbem, Mario Waespy, Olga Reichert, Philipp Mandel, Eric Drebitz, Frank Dietz
} and Sørge Kelm*

\begin{abstract}
Background: Animal African trypanosomiasis, sleeping sickness in humans and Nagana in cattle, is a resurgent disease in Africa caused by Trypanosoma parasites. Trans-sialidases expressed by trypanosomes play an important role in the infection cycle of insects and mammals. Whereas trans-sialidases of other trypanosomes like the American T. cruzi are well investigated, relatively little research has been done on these enzymes of $T$. congolense.

Results: Based on a partial sequence and an open reading frame in the WTSI database, DNA sequences encoding for eleven T. congolense trans-sialidase 1 variants with 96.3\% overall amino acid identity were amplified. Transsialidase 1 variants were expressed as recombinant proteins, isolated and assayed for trans-sialylation activity. The purified proteins produced $\alpha 2,3-$ sialyllactose from lactose by desialylating fetuin, clearly demonstrating their transsialidase activity. Using an HPLC-based assay, substrate specificities and kinetic parameters of two variants were characterized in detail indicating differences in substrate specificities for lactose, fetuin and synthetic substrates. Both enzymes were able to sialylate asialofetuin to an extent, which was sufficient to reconstitute binding sites for Siglec-4. A mass spectrometric analysis of the sialylation pattern of glycopeptides from fetuin revealed clear but generally similar changes in the sialylation pattern of the $\mathrm{N}$-glycans on fetuin catalyzed by the trans-sialidases investigated.
\end{abstract}

Conclusions: The identification and characterization of a trans-sialidase gene family of the African parasite $T$. congolense has opened new perspectives for investigating the biological role of these enzymes in Nagana and sleeping sickness. Based on this study it will be interesting to address the expression pattern of these genes and their activities in the different stages of the parasite in its infection cycle. Furthermore, these trans-sialidases have the biotechnological potential to be used for enzymatic modification of sialylated glycoconjugates.

\section{Background}

Animal African trypanosomiasis, called sleeping sickness in humans and Nagana in cattle, is a resurgent disease in Africa. Nagana is caused by Trypanosoma congolense (T. congolense), Trypanosoma vivax (T. vivax) and Trypanosoma brucei (T. brucei) subspecies. Most research on African trypanosomes has focused on T. brucei, whereas only few studies have been done with other African trypanosomes including T. congolense. In wild animals, these parasites cause relatively mild infections while in domestic animals they cause a severe, often

\footnotetext{
* Correspondence: skelm@uni-bremen.de

Centre for Biomolecular Interactions Bremen, Department of Biology and Chemistry, University of Bremen, Leobener Str./NW2/B2235, 28359 Bremen, Germany
}

\section{Biomed Central}

fatal disease. Because of Nagana, stock farming is very difficult within the tsetse belt of Africa [1].

Although of crucial importance for their survival, cyclical transmission and hence pathogenicity of trypanosomes, trypanosomes lack the biochemical metabolic machinery synthesizing sialic acids (Sia), but use a unique enzyme, trans-sialidase (TS) to transfer Sia onto the parasites surface molecules from the environment. Structurally TS belong to the family of sialidases (SA). In contrast to the usual sialyltransferases, TS does not utilize CMP-activated Sia as monosaccharide donors, but catalyzes the transfer of carbohydrate-linked Sia to another glycan forming a new $\alpha 2,3$-glycosidic linkage to galactose or $\mathrm{N}$-acetylgalactosamine.

Whereas more detailed studies exist on the role of TS in the pathogenicity of T. cruzi, the etiologic agent of 
Chagas diseases in South America, where TS was first discovered [2], the current knowledge about the corresponding enzymes in the African trypanosomes is very limited. Of all the African trypanosomes, only T. brucei full length TS genes have been cloned and studied [3]. Sialylation of parasite surfaces is believed to protect the parasites from the action of glycolytic enzymes as well as from immunocompetent substances present in the tsetse gut and blood meal respectively, as well as influencing the interaction of parasites with the gut epithelial cells [4-6]. In the African trypanosomes where TS is thought to be expressed only in the procyclic insect stages [5,7], TS has been shown to increase the survival, maturation and hence establishment of the parasites in the vector midgut [8].

Two TS forms, named TS-form 1 and TS-form 2, have been purified from procyclic $T$. congolense cultures [6]. Interestingly, glutamic acid and alanine-rich protein (GARP) was co-purified with TS-form 1, suggesting that GARP may be a natural substrate for TS-form 1. Interestingly, TS-form 1 had significantly less SA activity and higher TS activity, whereas SA activity was predominately found in preparations of TS-form 2. An anti-T. congolense TS antibody (mAb 7/23) was developed using TS form 1 as antigen. This antibody is specific for $T$. congolense TS recognizing TS-form 1 and TS-form 2, but does not bind to T. brucei TS. Peptides micro sequencing revealed the amino acid sequence VVDPTVVAK in TS-form 1. Subsequently, fragments of two TS genes (TS1 and TS2) were sequenced, sharing about 50\% sequence identity [9]. TS1 encoded this peptide sequence, whereas in TS2 this sequence ended in VVK. These data strongly suggested that the gene product of TS1 has been present in TS-form 1. Nevertheless, it has remained unclear whether only TS1 and/or TS2 gene products were present in TS-form 1 and TSform 2 preparations. Due to the fact that the monoclonal antibody mAb 7/23 bound both TS preparations, it is quite possible that TS-form 2 contained at least some amounts of TS1 gene product, which might have been responsible for the TS activity of this preparation.

Here, we report the cloning of eleven trans-sialidase TS1 variants from $T$. congolense and their recombinant expression in $\mathrm{CHO}_{\mathrm{Lec} 1}$ cells. Furthermore, the enzymatic properties of two of these recombinant TS1 variants were compared with TS from T. cruzi.

\section{Results}

\section{Diversity of TS1 genes and structural model}

Based on the partial sequence of TS1 [GenBank: AJ535487.1] [9], an open reading frame in the Welcome Trust Sanger Institute (WTSI) database was identified. The full-length translation product consists of 750 amino acids extending the partial sequence of TS1 by
153 amino acids at the $\mathrm{N}$-terminus and by 84 amino acids at the $\mathrm{C}$-terminus. It contains a 16 amino acids $\mathrm{N}$ terminal signal peptide and a catalytic domain (residues 17-467), which is connected through a long $\alpha$ helix (residues 468-491) to a lectin domain (residues 492-732) followed by a potential C-terminal GPI-anchor attachment site (residues 733-750, identified by big-PI predictor [10]). Furthermore, nine potential $N$-glycosylation sites were identified (Figure 1).

T. congolense TS1 shares about $57 \%$ sequence identity with T. brucei TS [EMBL: AAG32055.1] and 48\% with T. cruzi TS [EMBL: BAA09334.1] (Figure 2). The $T$. bruce $i$ TS has a prolonged N-terminus of approx. 90 amino acids, which is conserved in T. congolense TS1 sharing $50 \%$ amino acids, but is absent in T. cruzi TS. The catalytic domain of both African proteins has $60 \%$ and the lectin domain $43 \%$ sequence similarities. T. congolense TS1, like T. brucei TS and T. rangeli SA, has no C-terminal SAPA domain typical for T. cruzi TS [11]. Almost all amino acid residues reported to be required for TS activity are identical in TS1 with the exception of A325 (corresponding to P231 in T. cruzi TS) [11], R127, G344-Q346 and Y408 (corresponding to Y248 and W312, respectively in T. cruzi TS) [12] (Figure 2).

To produce recombinant protein for enzyme characterization, the DNA encoding amino acids 17-732 was amplified using genomic T. congolense DNA as a template and inserted into a mammalian expression vector as described in Methods. 13 clones were picked from two independent cloning experiments and sequenced. Interestingly, not all the 13 clones had identical sequences and eleven different sequences were obtained (TS1a through TS1j), exhibiting an overall amino acid identity of $96.3 \%$. A more detailed search of the WTSI database using these sequences as queries confirmed the presence of these TS1 genes in the T. congolense genome database. As shown in Figure 3, changes are not evenly distributed over the protein sequences. Eight were found in the lectin domain and 17 in the catalytic domain, some close to the predicted active site as shown in Figure 4A.

For a better understanding of how these differences may affect TS function, we calculated a model structure (Figure 4) for TS1 e-1 by homology modeling based on the crystal structure of T. cruzi TS [12], which was complexed with the Sia derivative 3-fluoro-5- $N$-acetyl-9-benzamido-2,9-dideoxy-neuraminic acid. The superimposed structures of T. cruzi TS and the T. congolense TS1 e-1 model had a root mean square deviation (RMSD) of $0.685 \AA$ over 594 aligned residues.

In Figure 4A, amino acids of the active site are highlighted. Most of the amino acids reported to be relevant for TS activity are identical in all T. congolense TS1 variants (white labels). However, differences to T. cruzi TS 


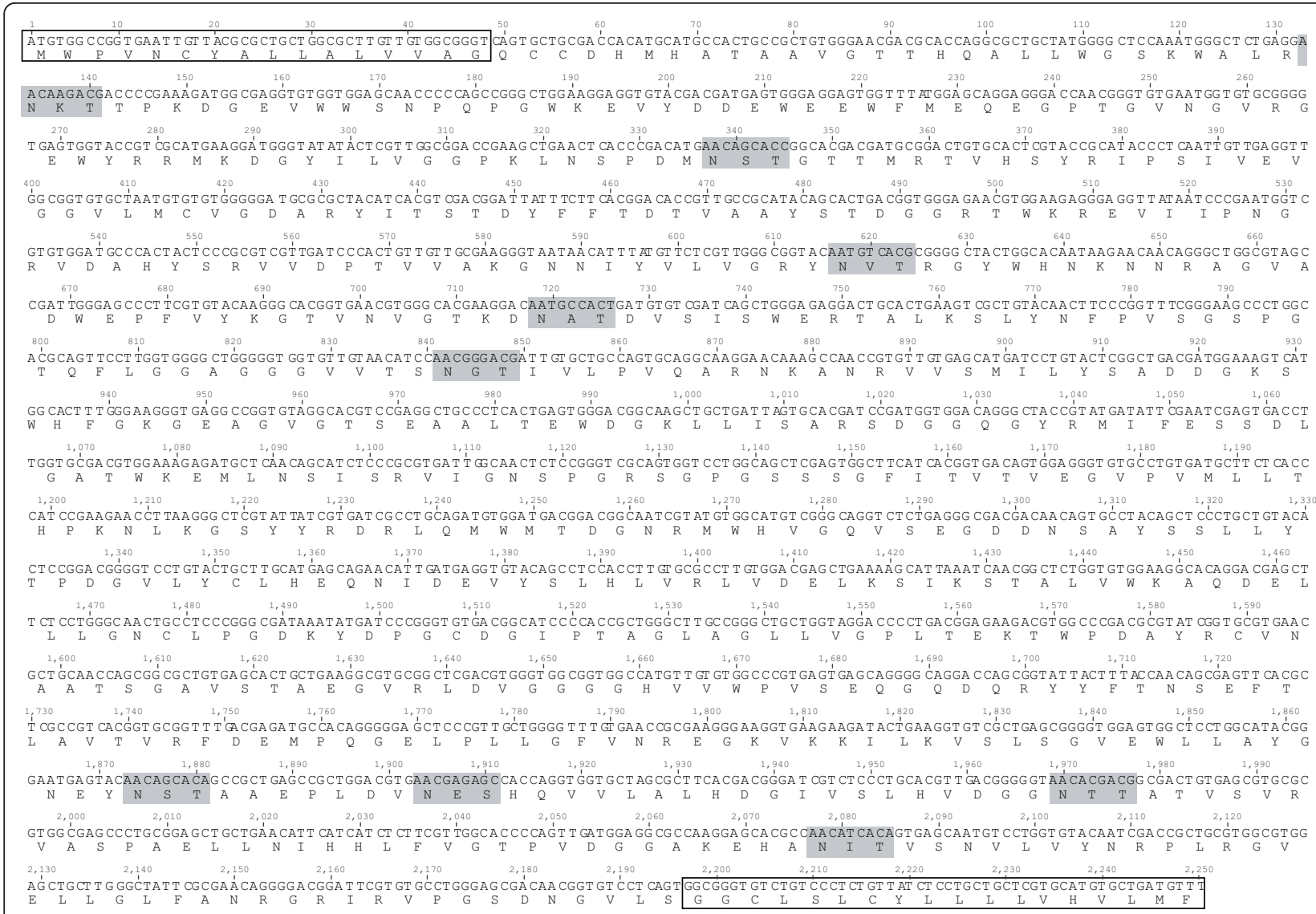

Figure 1 Primary sequence of TS1a. The full length coding domain sequence [EMBL: HE583283] with corresponding amino acid translations is shown. The recombinant protein was generated without the $\mathrm{N}$-terminal signal peptide and without the C-terminal region predicted to be replaced by a GPI anchor in the native protein (framed boxes). Predicted N-glycosylation sites are highlighted by light grey boxes.

were identified at three positions (yellow labels in Figure 4A). (I) At position 325 all T. congolense TS1 variants have an alanine, like in T. brucei TS, replacing a proline occurring in T. cruzi TS (P231); (II) Y408 of all T. congolense TS1 variants corresponds to a tryptophan in $T$. cruzi TS (W321) and T. brucei TS; (III) the group of G342, G343 and Q344 replaces a tyrosine (Y248) in $T$. cruzi TS. In addition, near the catalytic site at position 407 (red label) in T. congolense TS1 variants, a serine or valine occurs instead of arginine (R311) in T. cruzi TS. Interestingly, similar differences occur also in T. brucei TS (Figure 2). Since these amino acids are close to the active site, they could influence the acceptor binding specificity. The arginine at position 144 (blue label) is conserved in all TS, with the exception of $T$. congolense TS1g, where it is a cysteine.

In Figure 4B the amino acid positions are highlighted, which have different side chains in TS1a-TS1j (Figure 3 ). It should be noted that these are all on the same side of the protein as the catalytic site. Striking is a cluster of amino acid variations in the lectin domain (position 599 to 602 and 643) suggesting that these changes may influence substrate binding of larger substrate molecules, such as glycoproteins.

\section{Characterization of $T$. congolense TS1 enzyme activity}

All eleven TS1 gene products (TS1a-TS1j) were expressed as recombinant proteins and were recognized by the anti- $T$. congolense TS antibody ( $\mathrm{mAb} 7 / 23$ ) [6] (data not shown). For all TS1 variants similar robust TS activity could be determined, except for TS1g. This variant, which carries cysteine instead of arginine at position 144, had only very low TS activity. However, in contrast to the other variants, TS1g released free Sia from fetuin at about $50 \%$ of the transfer to lactose. Two of the T. congolense TS1 variants, TS1b and TS1 e-1, were further characterized. They differ in eleven of the total 25 positions with amino acid variations listed in Figure 3, three in the catalytic domain and eight in the lectin domain.

The donor substrates fetuin 3'SL or pNP-Neu5Ac and the acceptor substrates lactose, galactose or Gal-MU were employed to determine sialidase and trans-sialidase activities. For this purpose, a new assay was established 

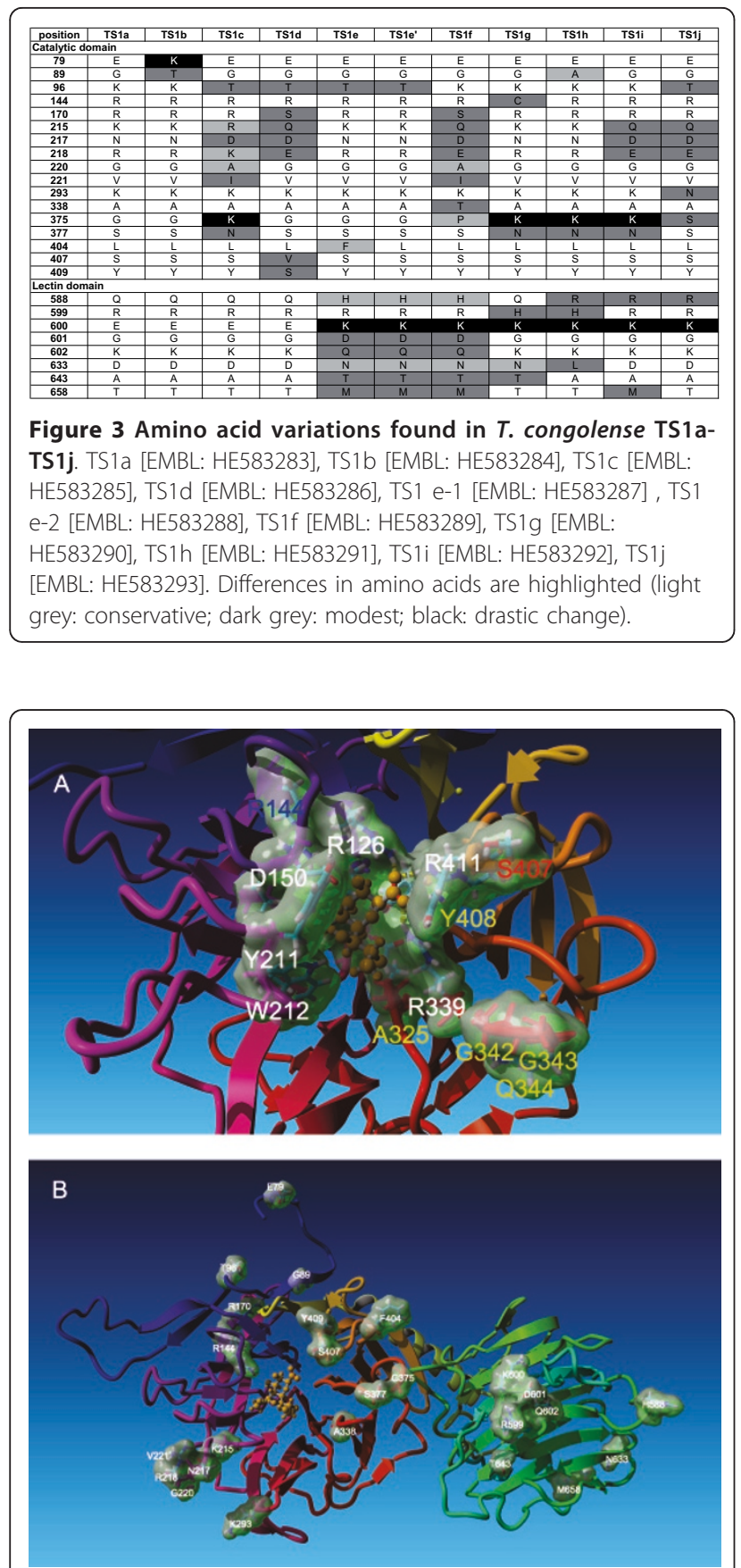

Figure 4 Homology model of $T$. congolense TS1. The crystal structure of T. cruzi TS [12] in complex with 3-fluoro-5- $\mathrm{N}$-acetyl-9benzamido-2,9-dideoxy-neuraminic acid was used as template to calculate a model structure for T. congolense TS1 e-1. Only the Neu5Ac part (orange) of the ligand in the binding site of the complex is illustrated. (A) Conserved amino acids of the active site are labeled in white. Amino acids at positions reported to be important for ligand binding in T. cruzi TS [12], which are not conserved in $T$. congolense are labeled in yellow. The red labeled position 407 is a serine or valine in T. congolense TS1 forms. R144, which is exchanged to a cysteine in TS1g, is labeled in blue. (B) Green clouds mark positions at which different amino acids occur in other T. congolense TS1 forms. as described under Methods, using HPAEC-PAD to quantify sialylated oligosaccharide products with the detection limit of $20 \mathrm{pmol}$ 3'SL corresponding to 0.5 $\mu \mathrm{M}$ in the reaction mixture. In standard assays, $50 \mu \mathrm{L}$ TS reactions were set up with 50 ng TS1b or TS1 e-1, $100 \mu \mathrm{g}$ fetuin (approx. $600 \mu \mathrm{M}$ bound Sia) as donor substrate and $100 \mathrm{nmol}$ acceptor substrate $(2 \mathrm{mM}$ e. g. lactose or galactose). Under these conditions, linear product formation was obtained for up to $2500 \mathrm{pmol}$ corresponding to $50 \mu \mathrm{M}$ 3'SL (Figure 5).

If lactose is used as a donor substrate under standard conditions, 3'SL concentration increases linearly for about 30 minutes before the reaction velocity started to decrease (Figure 5A). It should be noted that lactose was sialylated almost twice as fast by TS1b than by TS1 e-1. In contrast to lactose, galactose was sialylated at the same rate by both enzymes, but at about 20 -fold lower velocity than lactose.

Different specific activities were obtained for T. congolense TS1b, TS1 e-1 and T. cruzi TS (Figure 6). The reaction velocity was linearly dependent on the amount of TS as long as the concentration of the product 3'SL was below $50 \mu \mathrm{M}$. Under standard conditions $50 \mu \mathrm{M}$ 3'SL was produced in 30 minutes with $50 \mathrm{ng}$ TS. If 200 ng TS or more were used, product formation was independent of the amount of TS, probably due to the increased use of 3'SL as a donor substrate in the reverse reaction, finally leading to an equilibrium between lactose, 3'SL, sialylated and desialylated glycans on fetuin. This equilibrium apparently was reached in 30 minutes with $500 \mathrm{ng}$ TS $(266 \pm 4 \mu \mathrm{M}$ 3'SL for T. cruzi TS, 194 $\pm 6 \mu \mathrm{M}$ 3'SL for TS1b and $165 \pm 7 \mu \mathrm{M}$ 3'SL for TS1 e1). After $20 \mathrm{~h}$ incubation, $50 \mathrm{ng}$ TS was sufficient to reach the equilibrium. Independent of the amount of enzyme used, for all three TS applied similar final concentrations of 3'SL were obtained after $20 \mathrm{~h}$ incubation (Table 1).

The HPAEC-PAD method used allowed not only determining the TS, but also SA activity, since free Sia and 3' SL could be quantified from the same chromatogram. In standard reactions (50 ng TS, 30 min incubation time) no SA activity could be detected, both in the presence or absence of lactose as an acceptor substrate. This suggests that these TS1 variants usually need an acceptor substrate like lactose to cleave Sia from a donor substrate. However, after $20 \mathrm{~h}$ incubation, free Sia was detected. The quantity of Sia released was dependent on the amount of TS used (Table 1). Besides standard TS reactions with fetuin as donor and lactose as acceptor substrate, TS reactions with $2 \mathrm{mM}$ 3'SL as donor and $2 \mathrm{mg} / \mathrm{mL}$ ASF as acceptor substrate were performed. In these reactions, free Sia was detected after short reaction times and after incubation for $24 \mathrm{~h}, 0.5-1$ $\mathrm{mM}$ free Sia were produced (data not shown). 

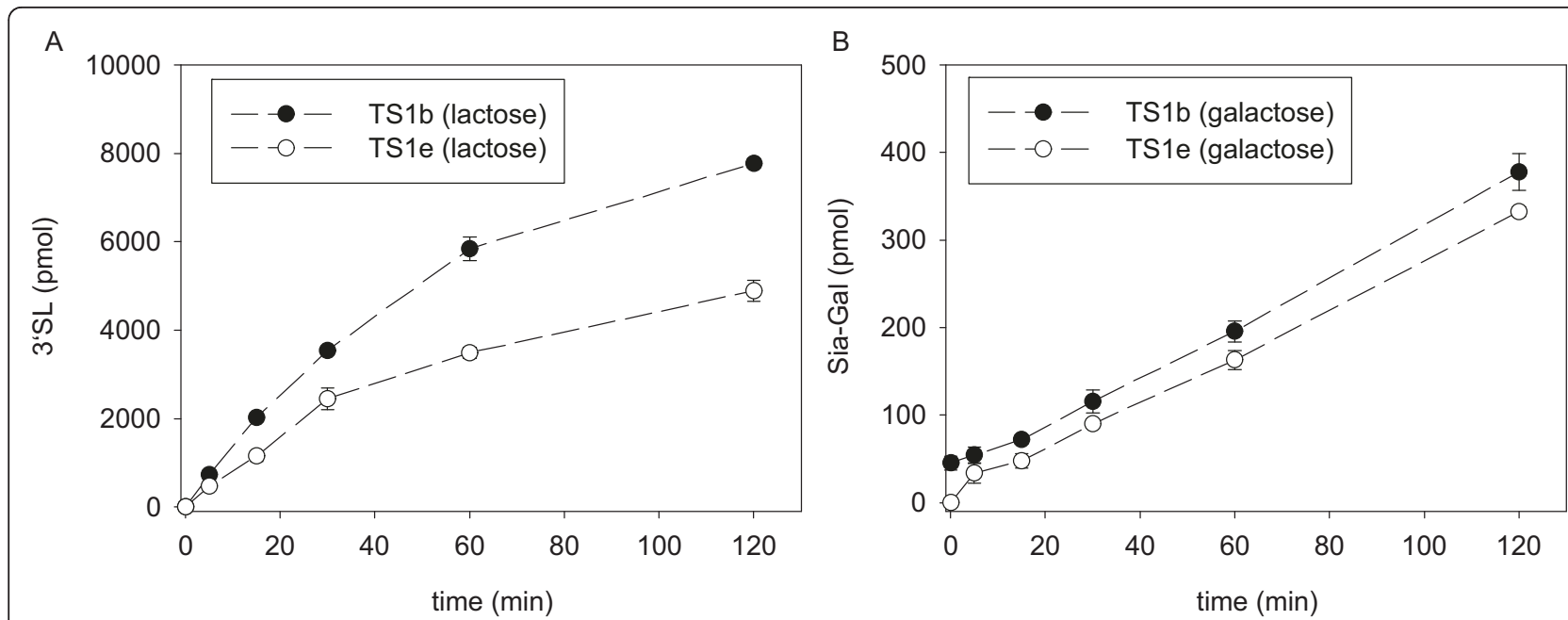

Figure 5 Time dependence of TS reactions. Reactions of $100 \mu \mathrm{g}$ fetuin with $100 \mathrm{nmol}$ lactose (A) or $100 \mathrm{nmol}$ galactose (B) were started with $50 \mathrm{ng}$ TS in $50 \mu \mathrm{L}$ and incubated at $37^{\circ} \mathrm{C}$. The amount of $3^{\prime} \mathrm{SL}$ produced was determined by HPAEC-PAD as described under Methods. Data points are means \pm standard deviations of three replicates.

For kinetic experiments, assays were incubated for 30 minutes using $50 \mathrm{ng}$ TS, since under these conditions 3'SL production was linear for all three TS. To determine the kinetic parameters for the acceptor substrates lactose (Figure 7A) or Gal-MU (Figure 7C), $100 \mu \mathrm{g}$ fetuin $(600 \mu \mathrm{M}$ bound Sia) was used as donor substrate. The lowest $\mathrm{K}_{\mathrm{M}}$ for lactose was found for T. cruzi TS with $327 \mu \mathrm{M}$ compared to $1683 \mu \mathrm{M}$ for TS1b and 727 $\mu \mathrm{M}$ for TS1 e-1 (Table 2). Furthermore, T. cruzi TS was able to produce twice more 3'SL than TS1b and fourfold more than TS1 e-1 under these conditions.

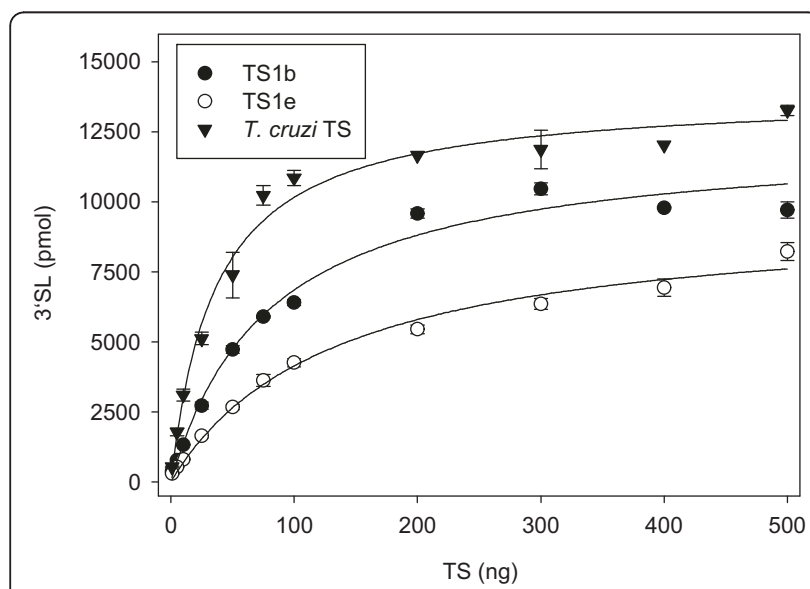

Figure 6 Concentration dependence of TS reactions. Reactions of $100 \mu \mathrm{g}$ fetuin with $100 \mathrm{nmol}$ lactose were started with varying amounts of TS in overall $50 \mu \mathrm{L} 10 \mathrm{mM}$ phosphate buffer, pH 7.4 and incubated at $37^{\circ} \mathrm{C}$ for $30 \mathrm{~min}$. The amount of $3^{\prime} \mathrm{SL}$ produced was determined by HPAEC-PAD as described under Methods. Data points are means \pm standard deviations of three determinations.
To determine the kinetic parameters for the donor substrate fetuin (Figure 7B), $2 \mathrm{mM}$ lactose was used as acceptor substrate. Both T. congolense TS1 had similar $\mathrm{v}_{\max }$-values, whereas the $\mathrm{v}_{\max }$ for $T$. cruzi TS was about fivefold higher. Different to the $K_{M}$ of lactose, the lowest $K_{M}$ for fetuin was exhibited by TS1b with $359 \mu \mathrm{M}$, which is about fivefold lower compared to TS1 e-1 with $1617 \mu \mathrm{M}$ and about 12-fold lower compared to T. cruzi TS with $4124 \mu \mathrm{M}$.

Kinetic studies with TS were also performed for the acceptor substrate Gal-MU (Table 2) and the donor substrate pNP-Neu5Ac. Almost similar $\mathrm{K}_{\mathrm{M}}$ and $\mathrm{v}_{\max }{ }^{-}$ values were found for both $T$. congolense TS1. The substrate pNP-Neu5Ac was only weakly used as a donor substrate by all three TS species. Therefore, no reliable kinetic parameters could be determined.

Table 1 Free Sia and 3'SL production at equilibrium of the reaction

\begin{tabular}{lcc}
\hline & Neu5Ac [pmol] & 3'SL [pmol] \\
\hline $\mathbf{0} \mathbf{n g}$ TS & $262 \pm 46$ & 0 \\
\hline $\mathbf{5 0} \mathbf{n g}$ T. cruzi TS & $580 \pm 8$ & $12597 \pm 115$ \\
\hline $\mathbf{2 5 0} \mathbf{n g}$ T. cruzi TS & $1820 \pm 13$ & $11199 \pm 288$ \\
\hline $\mathbf{5 0} \mathbf{n g}$ TS1b & $415 \pm 7$ & $15378 \pm 117$ \\
\hline $\mathbf{2 5 0} \mathbf{n g}$ TS1b & $1095 \pm 129$ & $14435 \pm 2226$ \\
\hline $\mathbf{5 0} \mathbf{n g}$ TS1 e-1 & $348 \pm 83$ & $12719 \pm 3057$ \\
\hline $\mathbf{2 5 0}$ ng TS1 e-1 & $1186 \pm 9$ & $13655 \pm 491$ \\
\hline only fetuin & $116 \pm 8$ & 0
\end{tabular}

Neu5Ac and 3'SL produced by TS after $20 \mathrm{~h}$ incubation under standard conditions were determined as described under Material and Methods. Data points are means \pm standard deviations of three replicates. 

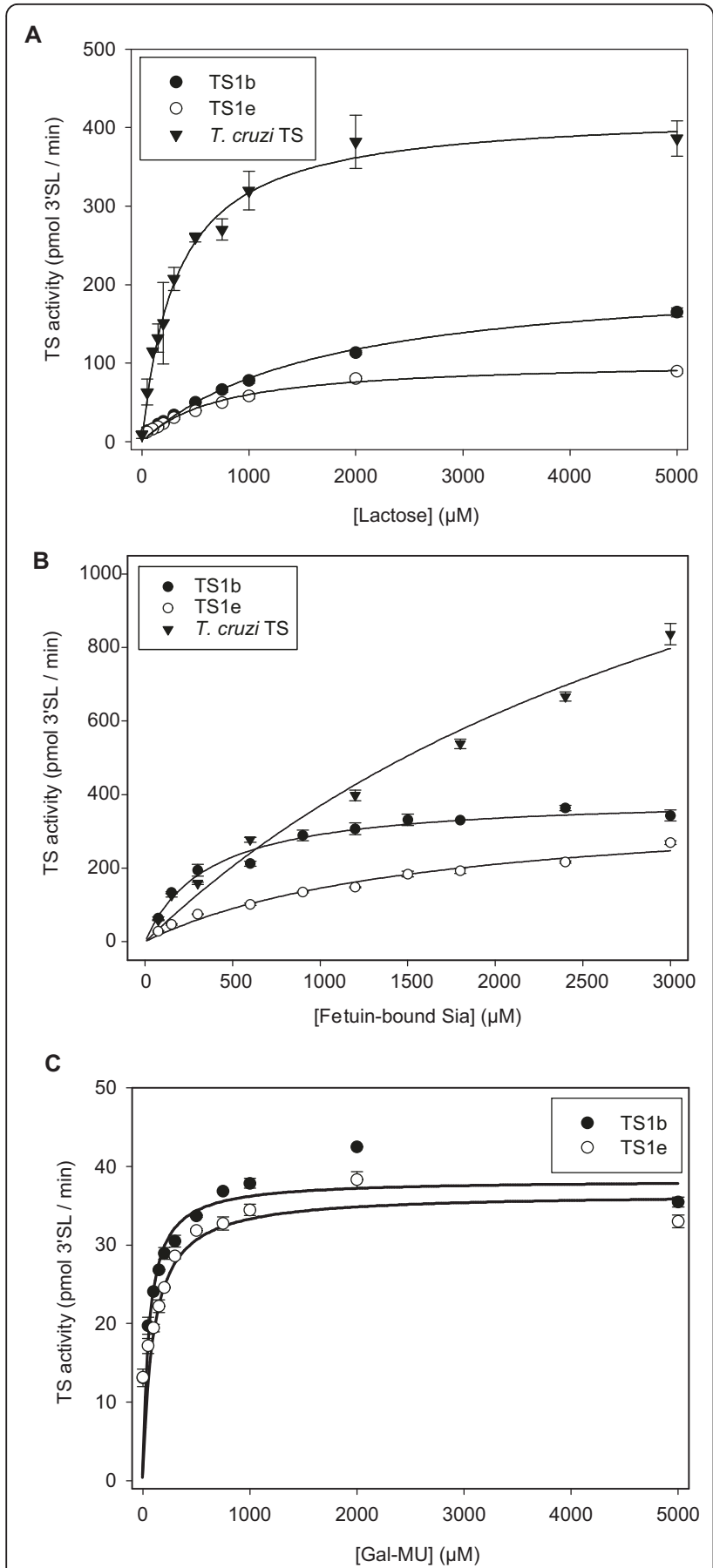

Figure $\mathbf{7}$ Michaelis-Menten kinetics of TS reactions. All reactions were started with $50 \mathrm{ng}$ TS in a final volume of $50 \mu \mathrm{L}$ and incubated for $30 \mathrm{~min}$ at $37^{\circ} \mathrm{C}$. (A) $600 \mu \mathrm{M}$ fetuin-bound Sia was used as donor substrate with varying concentrations of lactose as acceptor substrate. (B) Varying concentrations of fetuin-bound Sia were used as donor substrate with $2 \mathrm{mM}$ lactose as acceptor substrate. (C) Varying amounts Gal-MU were used as acceptor substrate and $50 \mathrm{nmol} 3^{\prime} \mathrm{SL}$ as donor substrate. The amount of 3'SL (in the case of $\mathbf{( A )}$ and $\mathbf{( B )}$ ) or $3^{\prime}$ Sia-Gal-MU (in the case of (C)) produced was determined by HPAEC-PAD as described under Methods. Data points are means \pm standard deviations of three replicates

\section{Sialylation pattern of glycopeptides}

Structural differences between TS1 variants may influence the enzymes preference for glycans on glycoproteins, such as fetuin. This could possibly result in different sialylation patterns on glycoproteins after incubation with TS. Fetuin contains $3 \mathrm{~N}$-glycosylation sites and $3 \mathrm{O}$-glycans, which all can serve as Sia donors in TS reactions [13]. To investigate the specificity of TS towards different $\mathrm{N}$-glycans on fetuin, we used MALDITOF-MS to determine the sialylation pattern of glycopeptides (GPs) from trypsin-digested fetuin after incubation with TS and lactose (Figure 8).

The sialylation patterns of three glycopeptides, GP 127-141 (dibranched or tribranched), and GP 54-85 (tribranched) were determined. All 14 potential sialylation variants of these glycopeptides could be identified unambiguously and quantified from the MALDI-TOFMS spectra. In untreated fetuin most branches on the three $\mathrm{N}$-glycans investigated were sialylated, whereas upon treatment with TS and lactose after 30 minutes a clear shift towards incompletely sialylated glycans was observed. After $24 \mathrm{~h}$ TS incubation the relative amounts of unsialylated glycans was further increased and monosialylated glycans represented the most prominent species on both, di- and tribranched glycans (Figure 9).

\section{Sialylation of glycoproteins}

As described above, T. congolense TS1b and TS1 e-1 readily used fetuin as donor substrate for the production of 3'SL. However, long-term TS reactions or experiments with higher amounts of TS had suggested that the reverse reaction also takes place. Therefore, we investigated whether $T$. congolense TS1b and TS1 e-1 can restore sialylated glycans on Vibrio cholerae sialidase-treated fetuin (ASF) as model glycoprotein. Resialylation experiments were performed with $100 \mu \mathrm{g}$ ASF as acceptor and $100 \mathrm{nmol} 3^{\prime} \mathrm{SL}$ as donor substrate as well as $50 \mathrm{ng}$ TS1 in $50 \mu \mathrm{L}$ to start the TS reaction and were incubated up to $24 \mathrm{~h}$. This resialylation partially reversed the shift in electrophoretic mobility in SDSPAGE observed for sialidase-treated fetuin (Figure 10). Also by MALDI-MS of glycopeptides, the sialylation of unsialylated glycans was confirmed (data not shown).

Furthermore, we addressed the question of whether through this reaction recognition sites for siglecs can be restored. For this purpose, TS-treated ASF was immobilized to a microtitre plate and used as target for Siglec4 , which preferentially binds $\alpha 2,3$-linked Sia. Under these conditions robust Siglec-4 binding was observed of ASF that had been treated with TS for $4 \mathrm{~h}$. A prolonged (up to $24 \mathrm{~h}$ ) TS reaction only led to little further increase reaching $40 \%$ of binding levels observed with native fetuin (Figure 11). 
Table 2 Kinetic parameters of $T$. cruzi TS, T. congolense TS1b and TS1 e-1

\begin{tabular}{|c|c|c|c|c|c|c|}
\hline & \multicolumn{4}{|c|}{ Acceptor substrates } & \multirow{2}{*}{\multicolumn{2}{|c|}{$\begin{array}{l}\text { Donor substrates } \\
\text { Fetuin-bound } \mathrm{Sia}^{\mathrm{x}}\end{array}$}} \\
\hline & \multicolumn{2}{|l|}{ Lactose } & \multicolumn{2}{|l|}{ Gal-MU } & & \\
\hline & $\begin{array}{c}\mathrm{V}_{\max } \\
(\mu \mathrm{mol} /(\min \times \mathrm{mg} \mathrm{TS}))\end{array}$ & $\begin{array}{c}\mathrm{K}_{\mathrm{M}} \\
(\mu \mathrm{M})\end{array}$ & $\begin{array}{c}\mathrm{V}_{\max } \\
(\mu \mathrm{mol} /(\min \times \mathrm{mg} \mathrm{TS}))\end{array}$ & $\begin{array}{c}K_{M} \\
(\mu M)\end{array}$ & $\begin{array}{c}\mathrm{v}_{\max } \\
(\mu \mathrm{mol} /(\min \times \mathrm{mg} \mathrm{TS}))\end{array}$ & $\begin{array}{c}K_{M} \\
(\mu M)\end{array}$ \\
\hline TS1b & $4.3 \pm 0.1$ & $1683 \pm 101$ & $0.77 \pm 0.03$ & $57 \pm 14$ & $7.9 \pm 0.3$ & $359 \pm 45$ \\
\hline TS1 e-1 & $2.1 \pm 0.1$ & $727 \pm 48$ & $0.72 \pm 0.03$ & $74 \pm 17$ & $7.6 \pm 0.5$ & $1617 \pm 223$ \\
\hline T. cruzi TS & $8.4 \pm 0.3$ & $327 \pm 31$ & n.d. & n.d. & $37.9 \pm 6.0$ & $4124 \pm 985$ \\
\hline
\end{tabular}

$\mathrm{K}_{\mathrm{M}}$ and $\mathrm{v}_{\max }$ were calculated from Michaelis-Menten kinetics (Figure 7) by SigmaPlot. Data points are means \pm standard deviations of three replicates. $\times$ Approximately $30 \mathrm{nmol}$ Sia per $100 \mu \mathrm{g}$ fetuin. Abbr.: n.d.: not determined.

\section{Discussion}

In 2003 Tiralongo et al. published a partial sequence for T. congolense TS1 [9]. Our approach to obtain the full length sequence of TS1 led to the discovery of 11 variants of this gene with an overall sequence identity of $96.3 \%$ in the genome of this parasite. The previously published partial TS1 sequence, which had been assembled from 47 independent PCR reactions, turned out to represent a mixture of fragments from the 11 TS1 forms identified in this study. Therefore, it is likely that that sequence doesn't exist in nature.

Similar TS-like gene families occur also in other trypanosomes. In $T$. brucei the situation appears to be less complex, since only 8 TS closely related genes have been identified [3] and these differences lead to 6 amino acid mutations. The largest TS gene family has been found in T. cruzi. Its 140 members fall into three different groups [14]. Blood stream trypomastigotes express two of these groups, one with TS activity and the other only with lectin activity. A third group has TS activity and is expressed by epimastigotes. At present it is unknown whether the expression of the different TS genes in African trypanosomes is also stage-dependent.
T. congolense TS1 shares about $57 \%$ identical amino acids with $T$. brucei $\mathrm{TS}$ and $48 \%$ sequence identity with T. cruzi TS. By comparison, the American T. rangeli SA and $T$. cruzi TS are more closely related with approximately $70 \%$ sequence identity [15].

The homology model for T. congolense TS1 based on crystal structures from T.cruzi TS and T. rangeli SA provided (I) insight in differences in the active site and its surrounding between TS from these parasites and (II) revealed the spatial distribution of the amino acid differences between the TS1 variants.

Compared to T. cruzi TS only three amino acids are changed in TS1 (A325, S407 and Y408). At position 325 a proline at the corresponding position of T. cruzi TS appears to be relevant for full TS activity [11]. However, in both, T. brucei TS and in T. congolense TS1, position 325 is an alanine. Tiralongo et al., (2003) postulated A325 might be common to African trypanosomes and does not seem to have an effect on enzymatic activity [9]. Our data have demonstrated that an alanine at this position is well compatible with TS activity, since all active TS1 variants have A325. The exchanges at 407 and 408 may be relevant for the different substrate

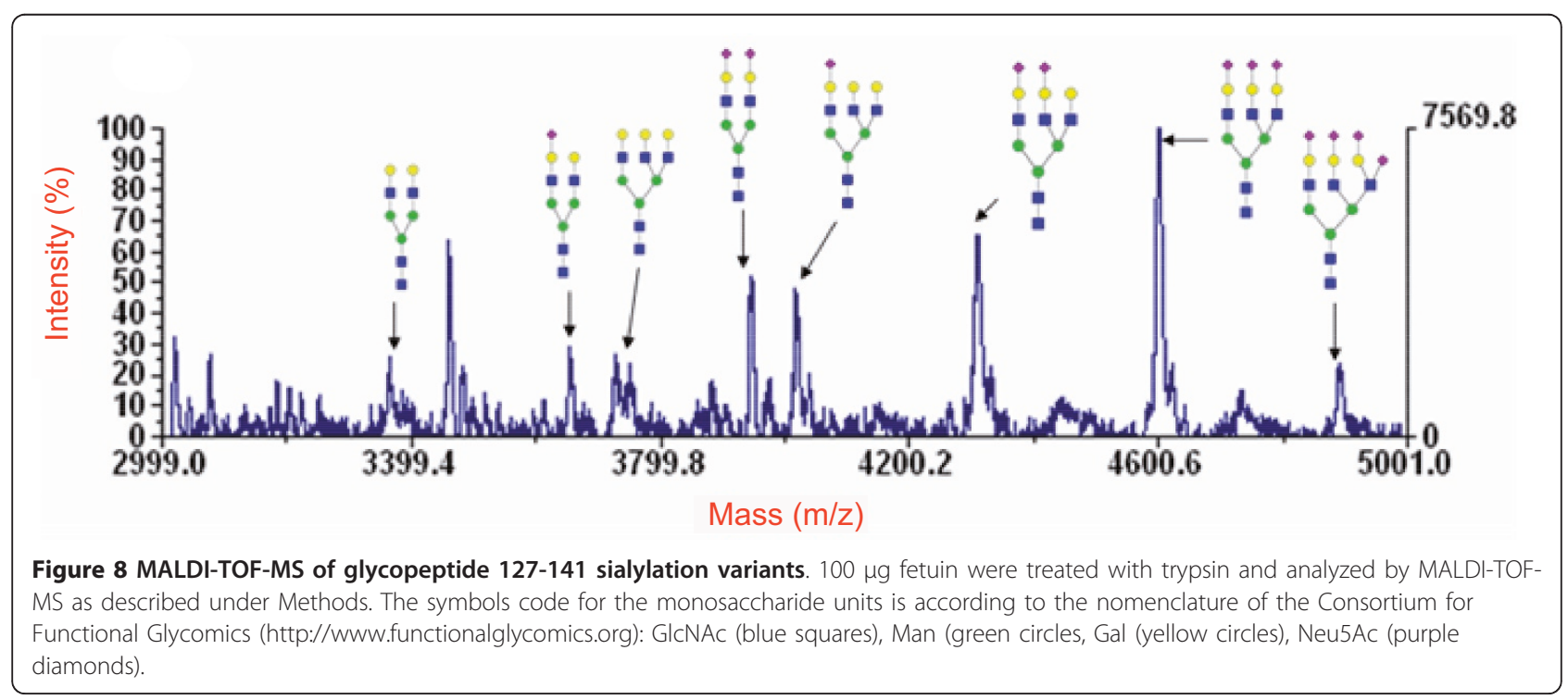




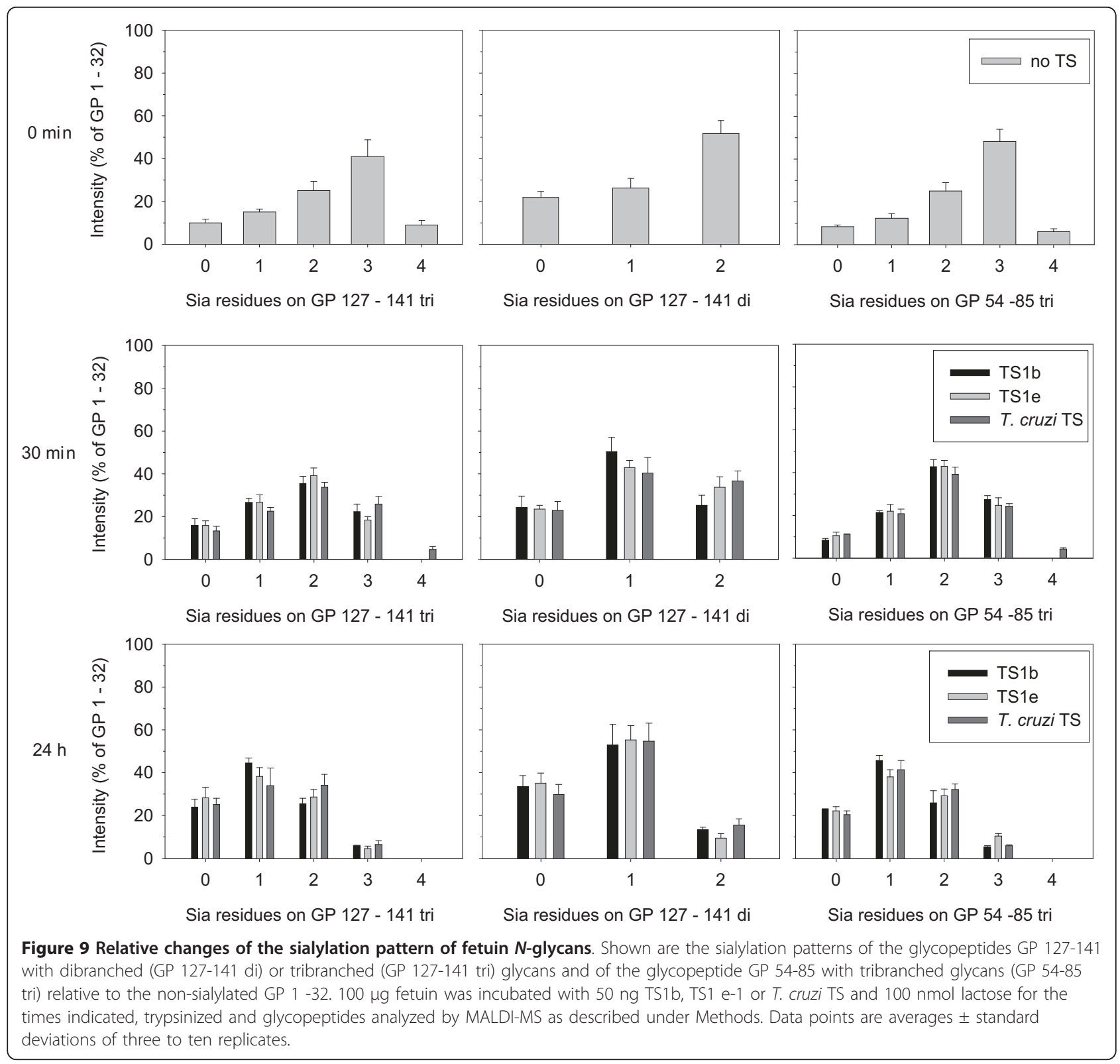

specificities of TS species, since this area possibly participates in the interaction with the underlying galactose.

With the full length coding sequence of TS1 available, the enzymes were expressed and characterized as recombinant proteins to address the question of whether TS1 accounts for the TS activity in the two preparations (TS-form 1 and TS-form 2) from procyclic parasite cultures [6]. The amino acid sequences of the eleven TS1 variants described here contain the peptide VVDPTVVAK present in TS-form 1. Furthermore, all recombinant TS1 variants reacted with the anti- $T$. congolense TS antibody (mAb 7/23) [6]. Based on this information, it is now clear that TS1 was present in both, TS-form 1 and TS-form 2. However, two observations made with recombinant TS1 suggest that the native enzyme preparations of TS-form 1 and even more for TS-form 2 contained additional TS1-like proteins. (I) The reaction velocities with the synthetic substrates pNP-Neu5Ac (as donor) and Gal-MU (as acceptor) were much lower than with fetuin and lactose for all three TS. Tiralongo et al., 2003 [6] determined a $K_{M}$ of $500 \mu \mathrm{M}$ for Gal-MU, which is approximately 10fold higher than for TS1b $(57 \mu \mathrm{M})$ and TS1 e-1 (74 $\mu \mathrm{M})$. These differences could be due to the presence of other TS1-like enzymes in the preparations of TS-form 1 and TS-form 2. (II) Using the substrate Neu5Ac-MU no SA or TS activity could be detected for the T. congolense TS1 variants investigated here, whereas cleavage of 


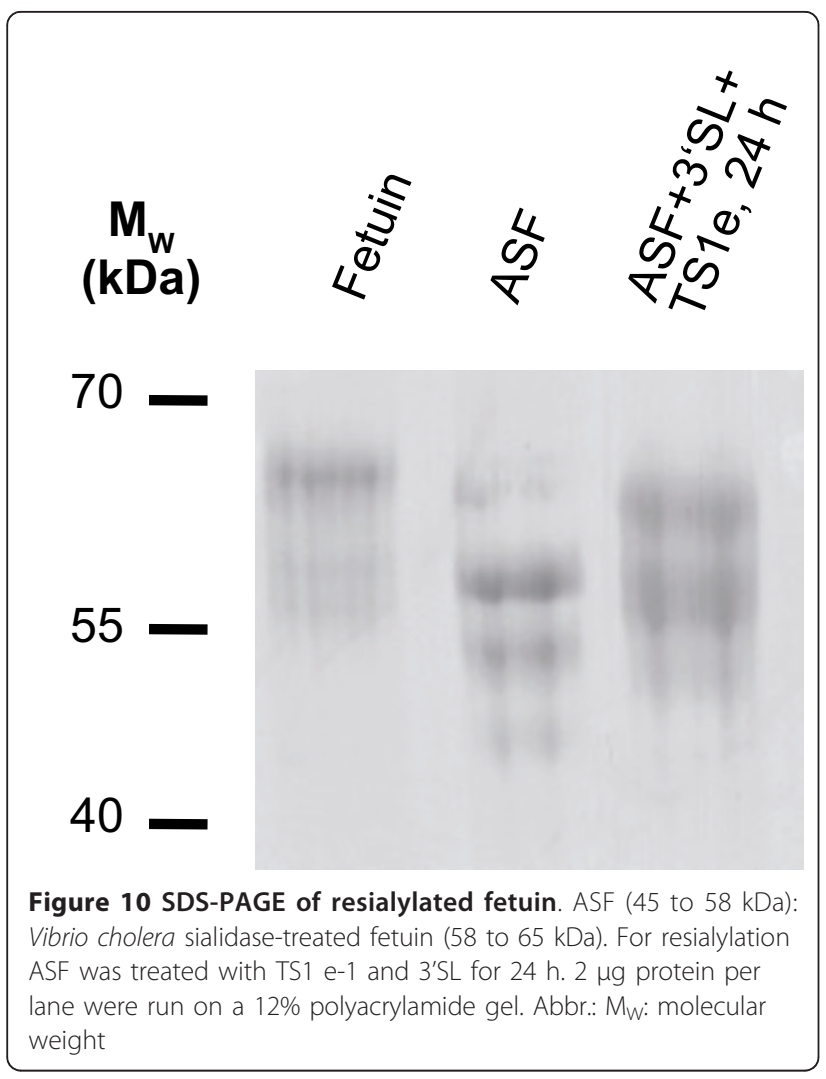

this substrate indicated SA activity in preparations of TS-form 1 and TS-form 2 [6], as well as TS activity in procyclic parasites [16]. Also this apparent discrepancy can easily be explained by the presence of other TS1like enzymes accepting Neu5Ac-MU as TS or SA substrate.

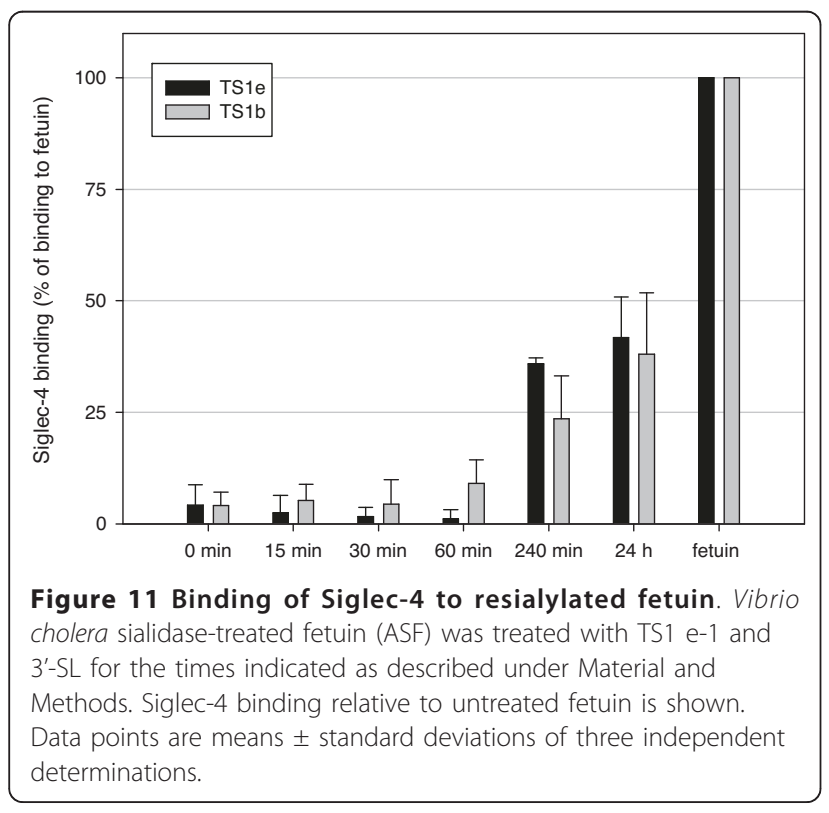

Ten of the eleven recombinant TS1 variants revealed TS activity, which is in agreement with the TS-form 1 purified by Tiralongo et al. [6]. Only TS1g, which carries a cysteine at position 144 instead of an arginine, showed very low TS but clear SA activity. This suggests that R144 is important but not essential for the transfer reaction and hinders SA activity in T. congolense TS1. In a previous study on T. rangeli SA [17] R144 has been postulated to form a hydrogen bond to the O4 of Sia. However, it remains unclear how this could relate to TS activity. Furthermore, the homology model of TS1 does not provide evidence for such a hydrogen bond for the binding site of $T$. congolense TS1.

The TS1 homology model (Figure 4B) displays also the position of amino acid exchanges in the eleven TS1 forms identified. It could be speculated that these positions are relevant determinants of antigenic sites and that these variations help to escape recognition by the immune system. However, if TS1 is expressed in the procyclic form in the insect vector, this is unlikely to be relevant. Interestingly, these exchanges are located on the same side of the protein where substrate binding occurs, which opens the possibility that these changes influence the binding specificities. The cluster of changes from position 599 to 602 and 643 could be involved in recognition of larger substrate molecules, such as glycoproteins. Positions, 407 and 408 (Figure 4B), as well as positions 375, 377 and 404 (Figure 4A) are closer to the active site in the catalytic domain, possibly contributing to interactions with substrates. It should be noted that the six amino acid differences occurring in T. brucei TS are not found in clusters like in $T$. congolense TS1, and furthermore, they are not even on the same side of the protein.

For a more detailed characterization of their differences in activity, we choose two TS1 variants, TS1b and TS1 e-1. The two amino acid sequences of TS1b and TS1 e-1 differ mainly in the lectin domain (Figure 4), whereby the exchange from E600 in TS1b to K600 in TS1 e-1 represents the most drastic change.

The observation that lactose is a much better acceptor substrate than $\mathrm{Gal}$ is in agreement with previously reported relative transfer activity data for the preparations TS-form 1 and TS-form 2 [9]. Interestingly, with fetuin as donor substrate T. cruzi TS, T. congolense TS1b and TS1 e-1 produced different amounts of 3'SL in 30 minutes under identical conditions. It is likely that these differences are due to distinct substrate specificities for the sialoglycans of fetuin. However, after $20 \mathrm{~h}$, equilibriums for the reactions were attained and the three TS applied produced almost the same amounts of 3'SL. Most likely this reflects a similar equilibrium for these three TS. 
Clear differences were found in the kinetic parameters of TS1b and TS1 e-1 for lactose and fetuin. Reliable kinetic parameter for 3'SL as donor substrate could not be determined, because of the inaccurate quantification of the high concentrations of 3'SL as donor substrate. Whereas TS1b has a slightly higher $K_{M}(1683 \mu \mathrm{M})$ for lactose compared to TS1 e-1 $(727 \mu \mathrm{M})$, the opposite and more pronounced difference was observed for fetuin-bound Sia, where TS1 e-1 has approximately fivefold higher $K_{M}(81 \mu \mathrm{M})$ compared to TS1b $(17 \mu \mathrm{M})$. In combination, this implies that the ratio of $K_{M}$ for lactose $/ K_{M}$ for fetuin-bound Sia is approximately 100 for TS1b, whereas it is only 10 for TS1 e-1. By comparison, for T. cruzi TS the $\mathrm{K}_{\mathrm{M}}$ for both substrates is quite similar $(326$ over $206 \mu \mathrm{M})$ and its $\mathrm{v}_{\max }$ was fourfold higher than for the TS1 isoforms. The differences in the kinetic parameters for fetuin observed for TS1b and TS1 e-1 are possibly related to altered affinities resulting from amino acid divergences in the lectin domain of TS1b and TS1 e-1. This would suggest a mechanism linking the lectin domain to the enzymatic properties of TS.

No release of free Sia could be detected after $30 \mathrm{~min}$ utes of TS reactions with fetuin as donor and lactose as acceptor substrates, demonstrating the absence of SA activity. However, after $20 \mathrm{~h}$ incubation, free Sia was detected clearly indicating SA activity. This activity correlated with the TS amount present, implying SA activity to be a side reaction observable only in extended reactions. Interestingly, in the reverse TS reactions with 3'SL as donor substrate for sialylation of galactose residues of ASF, free Sia is detected very early in the reaction (data not shown). This suggests that the free Sia detected in extended TS reactions times with fetuin as donor substrate is mainly the product of a SA side activity of the reaction using 3'SL as substrate. Therefore, the amount of free Sia could provide indirect information on the velocity of the reverse reaction. This assumption is further supported by the fact that lower amounts of TS can led to the same amount of final 3'SL but produce less free Sia as side product (Table 1). In this context it should be noted, that TS from T. cruzi clearly produced more free Sia than T. congolense TS1b or TS1 e-1. The structural basis for this phenomenon is unclear but may be related to the kinetics of the reaction. TS have been reported to follow ping-pong bi bi kinetics $[12,18,19]$. It will be interesting to investigate the structure-function relationship of this phenomenon and whether this is related to the SA activity of TS1g.

The TS substrate specificities for the glycans of the donor substrate fetuin were investigated by a MALDITOF analysis of TS treated glycopeptides from trypsin digested fetuin, since the glycosylation of fetuin is well established [13,20-23]. Three glycopeptides coming from two of the three $\mathrm{N}$-glycosylation sites (di- and tribranched GP 127-141, and tribranched GP 54-85) could be analyzed reliably. The predicted masses of GP 142-169 di could not be identified in any spectra. GP 54-85 di and GP 142-169 tri differed only in one Da, which were not resolved by the equipment available and were excluded from the analysis. The peptide containing the three $O$-glycosylation sites could not be detected due to its high mass. But it is important to note, that TS1 clearly utilizes sialylated $O$-glycans as donor substrates as indicated by a rapid unmasking of peanut agglutinin recognition sites (data not shown).

Only minor differences in the sialylation pattern of fetuin GPs were observed using the different TS species. In summary, these were too small to draw a conclusion that these TS differ in their substrate specificities for fetuin glycans. In general, the TS applied cleaved Sia from $N$-glycans of glycopeptides investigated, but also transferred Sia back to these $\mathrm{N}$-glycans. $\mathrm{N}$-glycans that carried three and four Sia molecules in the case of the tribranched $\mathrm{N}$-glycans as well as $\mathrm{N}$-glycans that carried two Sia molecules in the case of dibranched $N$-glycans before TS incubation, were reduced to predominantly 0 2 Sia molecules in the case of tribranched and 0-1 Sia molecule in the case of dibranched $N$-glycans after $24 \mathrm{~h}$ TS incubation.

Whereas in the reactions discussed above Sia was transferred from fetuin to synthesize 3'SL, we could also show that $T$. congolense TS1 as well as T. cruzi TS transfer Sia in the reverse direction from 3'SL to glycoproteins. Furthermore, the TS reaction restores binding of Sia-binding proteins, such as Siglec-4. Due to the reversibility of the reaction, a complete resialylation of an acceptor substrate applying TS cannot be expected under these conditions. Nevertheless, differences in the kinetic parameters as shown for two of the eleven $T$. congolense TS1 variants could be used in kinetically controlled reactions to optimize the TS reaction to one or the other product, making the TS1 variants interesting tools for biotechnological applications. Thus, TS1 can be utilized to transfer Sia in $\alpha 2,3$-linkage on biologically relevant glycoproteins containing terminal galactose as Sia acceptor.

\section{Conclusions}

For the first time, full length TS from the African parasite $T$. congolense has been cloned and sequenced, opening new perspectives for investigations on the biological role of these enzymes in the pursuit of a cure for Nagana and sleeping sickness. Eleven T. congolense TS1 variants were identified and expressed as recombinant proteins. The eleven TS1 differ in 25 of 702 amino acid positions and a structural model revealed that these variations occur in three clusters on the side of the protein that is open to substrate binding. Ten of these TS1 
variants share predominantly TS and little SA activity. Only one, TS1g, has much lower TS but increased SA activity, probably due to an exchange of an arginine to a cysteine at position 144. Interestingly, the kinetic parameters of two characterized TS1 variants reveal subtle differences in substrate specificities. However, these did not lead to major differences in the sialylation pattern of $\mathrm{N}$-glycans on fetuin after treatment with different TS variants. Finally a proof of principle has been provided that these TS can be used to sialylate glycoconjugates to create binding sites for Sia-binding proteins like Siglec-4.

It will be interesting to investigate the expression patterns of TS1 variants in the parasite's life cycle in future investigations addressing their importance for the manifestation of midgut colonization and maturation in the tsetse vector with possible implications for the transmission to the mammalian host.

\section{Methods \\ Materials}

Complete Mini, EDTA free protease-inhibitor tablets and Vibrio cholerae sialidase were purchased from Roche Diagnostics, Mannheim, Germany. Pfu DNA polymerase and restriction enzymes BamHI and SpeI, isopropyl $\beta$-D-1-thiogalacto-pyranoside (IPTG), PageBlue and molecular weight marker (PageRuler) were from Fermentas, St. Leon-Rot, Germany. Trypsin was from Promega, Mannheim, Germany, 2,5-Dihydroxybenzoic acid from Bruker Daltonics, Billerica, USA. Ultrafiltration units Vivaspin6 and VivaCell250 were from Sartorius, Göttingen, Germany. BCA Protein Assay Kit was purchased from Thermo Scientific Pierce, Rockford, USA. Anti-SNAP-tag rabbit polyclonal antibody was from GenScript, Piscataway, USA. Anti-Strep-tag rabbit polyclonal antibody, StrepTactin beads and buffers were purchased from IBA, Göttingen, Germany and hygromycin from PAA, Pasching, Austria. 2'-(4-Methylumbelliferyl) $\alpha-D-N$-acetylneuraminic acid sodium salt hydrate (MU-Neu5Ac), 4-methylumbelliferyl $B$-D-galactoside (MU-Gal), 4-methylumbelliferone (MU), glucoronic acid, Ex-cell ${ }^{\circledR}$ CD CHO media, fetuin and PEI transfection reagent were purchased from Sigma-Aldrich, Munich, Germany. X-ray film, enhanced chemiluminescence system, Ni-NTA and Q-Sepharose FF were purchased from GE Healthcare, Munich, polyvinylidene difluoride membranes and ZipTips from Millipore, Schwalbach, Germany.

\section{Cloning and expression of recombinant TS1}

The published partial sequences of Trypanosoma congolense TS1 [Genebank: AJ535487.1] [6,9] was used as starting query for searching the T. congolense genomic database for pathogen genomics at the WTSI (http:// www.sanger.ac.uk/). Based on the obtained sequence fragments, an open reading frame encoding TS1 was assembled. Based on this, primers were designed to amplify TS1 from T. congolense (strain STIB 249) genomic DNA [9] using Pfu DNA polymerase in a nested PCR reaction leaving out the $\mathrm{N}$-terminal signal peptide sequence and the C-terminal GPI anchor attachment site. Both outside primers (forward ATG CGG CCG GTG AAT TGT TAN and reverse CAT CAG CAC ATG CAC GAG CAN) were degenerate at the 3' end, whereas the internal primers (forward CGA CTA GTC AGT GCT GCG ACC ACA TGC AN and reverse CGG GAT CCG TCG CTC CCA GGC ACA CGA AN) were designed to introduce Spe 1 and $B a m H I$ restriction sites, respectively. These restriction sites were used to ligate the PCR-products in frame into a modified pDEF [24] vector ( $\mathrm{pDEF}-\mathrm{T} 3 \mathrm{C} / \mathrm{SNAPstrep}$ ) providing in frame a Transin cleavable signal peptide [25], a 3C-protease cleavage site [26] followed by SNAP (Covalys, Witterswil, Switzerland) and Strep (IBA, Göttingen, Germany) tags. The pDEF-T3C/SNAPstrep was obtained as follows: The coding sequence for the Transin signal peptide has been introduced into pcDNA 3 Amp Strep-tag [27] using the HindIII/BamHI-digested linker obtained by hybridization of the following oligonucleotides: sense 5'-CGAAGCTTATGAAAGGGCTCCCAGTCCTGC TGTGGCTGTGTACGGCTGTGTGCTC

ATCCTACCCATTGCATGGCAGTGAAGAAGAT GCTGGCATGGAGACTAGTGGATCCCG

and antisense primer 5'-

CGGGATCCACTAGTCTCCATGCCAGCATCTT CTTCACTGCCATGCAATGGGTAGG

ATGAGCACACACAGCCGTACACAGCCACAGCA GGACTGGGAGCCCTTTCATAAGCTTCG.

This Transin linker introduced a unique SpeI restriction site. The coding sequence of the hAGT protein (SNAP) was amplified using the pSNAP-tag ${ }^{\circledR}$ (T7) vector (NEB, Ipswich, MA, USA) as a template and subcloned in frame into BamHI/EcoRI digested pcDNA3 Amp Transin Strep-tag providing pcDNA3-T3C/SNAPstrep. The primers used for amplification were: sense 5'CGGGATCCCTGGAGGTGCTGTTCCAGGGCCCCATGGACAAAGACTGCGAAATGAAGCG-3' including the coding sequence for the $3 \mathrm{C}$ protease recognition site of the human rhinovirus HRV 3C (LEVLFQGP, underlined) and antisense 5'- CGGAATTCACCCAGC CCAGGCTTGCCCAGA.

$\mathrm{CHO}_{\mathrm{Lec} 1}$ cells were used for TS1 expression due to their ability to express only high-mannose glycans, since these cells are lacking $N$-acetyl glucosaminyltransferase I [28]. Transfection of $\mathrm{CHO}_{\text {Lec1 }}$ cells grown in aMEM supplemented with $10 \%$ fetal calf serum at $37^{\circ} \mathrm{C}, 5 \%$ $\mathrm{CO}_{2}$ was accomplished with polyethylenimine (PEI) transfection reagent following the manufacturer's 
instructions. 24 h after transfection, cells were passaged into 96-well plates in a selection media of $\alpha$ MEM containing varying amounts of hygromycin, ranging from $400 \mu \mathrm{g} / \mathrm{mL}$ to $1000 \mu \mathrm{g} / \mathrm{mL}$. Expression of recombinant TS1 (120 kDa including SNAP and Strep tags) was tested by analyzing cell culture supernatant using Western blots with anti TS1 monoclonal antibody (mAb 7/ 23) as primary antibody [9]. The presence of SNAP and Strep tags was confirmed using anti-SNAP-tag rabbit polyclonal antibody and anti-Strep-tag rabbit polyclonal antibody respectively in Western blots analysis. Selected cells were then adapted to Ex-cell ${ }^{\circledR} \mathrm{CD}$ CHO media supplemented with $8 \mathrm{mM}$ L-glutamine.

\section{Purification of Trypanosoma congolense trans-sialidase}

The harvested tissue culture supernatant was supplemented with $10 \mathrm{mM}$ Tris/ $\mathrm{HCl}, \mathrm{pH}$ 7.5, $1 \mathrm{mM}$ EDTA, 1 mM DTT and $0.02 \%$ sodium azide (all final concentrations) and centrifuged at $125,000 \times \mathrm{g}$ for $1 \mathrm{~h}$. The cleared supernatant was then concentrated 100 -fold by ultrafiltration (100 kDa cut off). Buffer was exchanged twice using $250 \mathrm{~mL} 100 \mathrm{mM}$ Tris-Cl, $\mathrm{pH} 8.0,150 \mathrm{mM}$ $\mathrm{NaCl}, 1 \mathrm{mM}$ EDTA (buffer A) in the same ultrafiltration unit and concentrated to a total volume of $10 \mathrm{~mL}$ for 1 $\mathrm{L}$ tissue culture supernatant. This was further clarified by centrifugation at $21,000 \times \mathrm{g}$ for 30 minutes before applying on a column of $1 \mathrm{~mL}$ Strep Tactin ${ }^{\circledR}$ beads equilibrated with buffer A. After loading the column was washed with 5 column volumes wash buffer $(100 \mathrm{mM}$ Tris-Cl, pH 8.0, $150 \mathrm{mM} \mathrm{NaCl}, 1 \mathrm{mM}$ EDTA) and TS1 was eluted with 3 column volumes of elution buffer (100 mM Tris-Cl, pH 8.0, $150 \mathrm{mM} \mathrm{NaCl}, 1 \mathrm{mM}$ EDTA, $2.5 \mathrm{mM}$ desthiobiotin) in fractions of $0.5 \mathrm{~mL}$. The affinity purified TS1 was dialyzed (10 kDa cut off) against $10 \mathrm{mM}$ phosphate, $\mathrm{pH}$ 7.4. Purification products were analyzed by SDS-PAGE and quantified by BCA assay with bovine serum albumin as standard.

\section{Expression and purification of Trypanosoma cruzi trans- sialidase}

Recombinant T. cruzi TS was produced in E. coli M15 (pREP4) according to Agusti et al., 2004 and Neubacher et al., $2005[29,30]$. In brief, cells were grown in $1 \mathrm{~L}$ "terrific broth" medium overnight at $18^{\circ} \mathrm{C}$. Protein expression was initiated with $0.5 \mathrm{mM}$ IPTG. The cells were dissolved in $40 \mathrm{~mL}$ lysis buffer $(50 \mathrm{mM}$ phosphate, $300 \mathrm{mM} \mathrm{NaCl}, \mathrm{pH} 8.0$ and $0.05 \%$ Lubrol), 1 tablet protease-inhibitor (Complete Mini, EDTA free) and 1 spatula tip of lysozyme were before incubation of 30 minutes at $4^{\circ} \mathrm{C}$. The cells were disrupted by 5 cycles of sonification on ice. Cells debris was removed by centrifugation at $40,000 \times \mathrm{g}$ for 60 minutes at $4^{\circ} \mathrm{C}$ and the supernatant was filtered using a $0.2 \mu \mathrm{m}$ pore size filter. $20 \mathrm{mM}$ imidazol was added before application on 0.5
mL Ni-NTA beads. Target proteins were eluted in the same buffer and $250 \mathrm{mM}$ imidazole. The eluted protein was dialyzed against $20 \mathrm{mM}$ Tris, $30 \mathrm{mM} \mathrm{NaCl}, \mathrm{pH} 8.0$ and further purified using a Q-Sepharose FF column in the same buffer with a linear gradient up to $1 \mathrm{M} \mathrm{NaCl}$. The activity of the purified protein was tested by a sialidase activity assay using MU-Neu5Ac as donor substrate and lactose as acceptor substrate as described below.

\section{Vibrio cholerae sialidase treatment of fetuin}

Asialofetuin (ASF) was prepared from fetuin by Vibrio cholerae sialidase (VCS) treatment as described [31]. In brief, fetuin was digested with VCS in $50 \mathrm{mM}$ sodium acetate, $9 \mathrm{mM} \mathrm{CaCl}_{2}$, pH 5.5 overnight at $37^{\circ} \mathrm{C}$ in a dialysis bag against the same buffer and afterwards against distilled water. Sialylated fetuin and sialidase was separated from ASF by anion exchange chromatography using Q-Sepharose. The proteins were eluted by a linear gradient from 0 to $1 \mathrm{M} \mathrm{NaCl}$ in $10 \mathrm{mM}$ Tris, $\mathrm{pH}$ 7.4. Collected fractions were assayed for protein at $280 \mathrm{~nm}$ and for SA activity with MU-Neu5Ac acid as substrate as described below. The fractions containing ASF but no SA activity were pooled and the buffer was exchanged against $10 \mathrm{mM}$ phosphate, $\mathrm{pH} 7.4$ using VivaSpin6 ultrafiltration units (10 kDa cut off).

\section{Sialidase activity assay}

Sialidase activity (+/- lactose) was determined by applying a microtitre plate assay detecting free 4-methylumbelliferone (MU) released from Neu5Ac-MU [6]. In brief, $50 \mu \mathrm{L}$ sample were incubated with $1 \mathrm{mM}$ Neu5Ac-MU (final concentration) in a black 96-well microtitre plate. To determine T. cruzi TS $1 \mathrm{mM}$ lactose was added as acceptor substrate. The plate was centrifuged for 1 minute at $1,000 \times \mathrm{g}$ and incubated for 30 minutes at room temperature in the dark. The reactions were stopped with $200 \mu \mathrm{L} 100 \mathrm{mM}$ glycine, $\mathrm{pH} 10$ and the fluorescence intensities were measured at $355 \mathrm{~nm}$ excitation and $460 \mathrm{~nm}$ emission using a fluorimeter (Ascent Fluoroscan).

\section{Trans-sialidase reactions}

The principle of this assay is based on the quantification of sialylated oligosaccharides by high performance anion-exchange chromatography with pulsed amperometric detection (HPAEC-PAD) as described below. In these reactions either fetuin is used as donor substrate, e.g. lactose as acceptor or 3'-sialyllactose (3'SL) is used as donor substrate with ASF as acceptor. A final volume of $50 \mu \mathrm{L} 10 \mathrm{mM}$ phosphate buffer were used for all TS reactions. Stock solutions of donor and acceptor substrate were mixed in $40 \mu \mathrm{L}$ buffer $(10 \mathrm{mM}$ Tris/HCl, $\mathrm{pH}$ 7.5) and the reactions were started with $10 \mu \mathrm{L}$ TS (50 ng in standard reactions) and incubated at $37^{\circ} \mathrm{C}$. The 
reactions were stopped with $200 \mu \mathrm{L}$ ice-cold acetone containing $28 \mu \mathrm{M}$ glucuronic acid and incubated overnight at $-20^{\circ} \mathrm{C}$. After centrifugation for 15 minutes at $20,000 \times \mathrm{g}$ and $4^{\circ} \mathrm{C}, 225 \mu \mathrm{L}$ supernatant were removed and both, protein pellets and supernatants were lyophilized.

The dried supernatant of the acetone precipitation was dissolved in $125 \mu \mathrm{L} \mathrm{H}_{2} \mathrm{O}$ for HPAEC-PAD, which was carried out by using a DX600 system (Dionex, Sunnyvale, CA, USA) with an electrochemical detector (ED50), a gradient pump (GP50) and an autosampler (AS50). Carbohydrates were separated by HPAEC on a CarboPAC PA1 $(4 \times 250 \mathrm{~mm})$ analytical column (Dionex) together with a guard column $(4 \times 50 \mathrm{~mm})$ using a constant flow rate of $1 \mathrm{~mL} / \mathrm{min}$. Sample volumes of $25 \mu \mathrm{L}$ were injected and the chromatography was performed as follows: $100 \mathrm{mM} \mathrm{NaOH}$ for $2 \mathrm{~min}$, followed by 100 $\mathrm{mM} \mathrm{NaOH} / 100 \mathrm{mM} \mathrm{NaOAc}$ for $22 \mathrm{~min}$. The column was regenerated by washing for 5 min with $100 \mathrm{mM}$ $\mathrm{NaOH} / 500 \mathrm{mM} \mathrm{NaOAc}$, followed by 5 min with 100 $\mathrm{mM} \mathrm{NaOH}$. For PAD the typical quadruple waveform was used as described previously [32]. The Dionex software Chromeleon 6.40 SP8 was used for data acquisition and data evaluation.

\section{Calculation of kinetic parameters}

$\mathrm{V}_{\max }$ and $\mathrm{K}_{\mathrm{M}}$ were calculated using the curve fit module of SigmaPlot 11 employing the Michaelis-Menten equation $\mathrm{v}=\mathrm{v}_{\max } \times \mathrm{c}_{\mathrm{s}} /\left(\mathrm{c}_{\mathrm{s}}+\mathrm{K}_{\mathrm{M}}\right)$.

\section{Siglec-4 binding assay}

Murine Siglec- $4_{\mathrm{d} 1-3}-\mathrm{Fc}$ was purified by protein-A affinity chromatography from tissue culture supernatants of stably transfected CHO Lec3.2.8.1 as described before [33]. The protein solution was dialyzed against $10 \mathrm{mM}$ phosphate buffer $\mathrm{pH} 7.4$, sterile filtered and stored at $4^{\circ} \mathrm{C}$. Binding assays with Siglec-4 were performed as described previously [33]. In brief, $4 \mu \mathrm{g} / \mathrm{mL}$ fetuin, ASF or TS-treated fetuin were immobilized in microtitre plates and binding of serially diluted Siglec- $4_{\mathrm{d} 1-3}-\mathrm{Fc}(8$ dilutions starting with $16 \mu \mathrm{g} / \mathrm{mL}$ ) was determined using alkaline phosphatase-labeled anti-Fc antibodies. The concentrations sufficient for $50 \%$ binding (relative to Siglec- $4_{\mathrm{d} 1-3}-$ Fc binding to fetuin) were determined from corresponding binding curves. At least three independent titrations were performed.

\section{SDS-PAGE and Western Blot analysis}

Samples were separated by SDS-PAGE (MiniProtean III; Bio-Rad, München, Germany) according to Laemmli [34] and stained with PageBlue.

For Western blot analysis, samples were transferred onto polyvinylidene difluoride membranes after SDS-PAGE. The membranes were blocked with 5\% BSA in Tris- buffered saline (TBS) buffer containing 0.15\% Tween 20 (TBS-T) for $1 \mathrm{~h}$. Washing of the membrane was done five times for 5 minutes each using TBS-T. Immunodetection was performed by incubating membranes with a primary antibody diluted in blocking buffer overnight at $4^{\circ} \mathrm{C}$. The following antibodies were used: anti- $T$. congolense TS mAb 7/23 (1:1000) and rabbit anti-Strep-tag (1:1000). Following four washes with TBS-T of 10 minutes each, the membranes were incubated with a secondary antibody conjugated to horseradish peroxidase for $2 \mathrm{~h}$ at room temperature. After washing four times with TBS- $\mathrm{T}$, blots were developed with the enhanced chemiluminescence system using X-ray film.

\section{Matrix-assisted laser desorption ionisation-time of flight mass spectrometry (MALDI-TOF-MS)}

TS reactions were carried out with $50 \mathrm{ng}$ TS, $100 \mathrm{nmol}$ lactose and $100 \mu \mathrm{g}$ fetuin as described above. The dried protein pellets after TS reaction were dissolved in $200 \mu \mathrm{L}$ $50 \mathrm{mM}$ ammonium hydrogen carbonate, $\mathrm{pH} 7.8$ and 1.6 $\mu \mathrm{L} 45 \mathrm{mM}$ DTT were added. After 30 minutes incubation at $50^{\circ} \mathrm{C}, 1.6 \mu \mathrm{L} 100 \mathrm{mM}$ IAA were added and further incubated for 30 minutes at $37^{\circ} \mathrm{C}$. The tryptic digestion was started with $2 \mu \mathrm{g}$ trypsin dissolved in $1 \mu \mathrm{L} 50 \mathrm{mM}$ acetic acid and incubated overnight at $37^{\circ} \mathrm{C}$ and stored at $-20^{\circ} \mathrm{C}$.

$5 \mu \mathrm{L}$ of the tryptic digest were mixed with $5 \mu \mathrm{L} \mathrm{H}_{2} \mathrm{O}$ and $1 \mu \mathrm{L} 1 \%$ trifluoroacetic acid and were directly mixed with matrix and $1 \mu \mathrm{L}$ was applied to the MALDITOF-MS target plate. The remaining $9 \mu \mathrm{L}$ were desalted using C18-reversed phase pipette tips (ZipTip). Peptides were eluted with $3 \times 100 \mu \mathrm{L} 12 \%$ and $30 \%$ acetonitrile in $\mathrm{H}_{2} \mathrm{O}$ and lyophilized. 2,5-Dihydroxybenzoic acid in $0.1 \%$ trifluoroacetic acid was used as MALDI matrix. The dried peptides were directly dissolved in $10 \mu \mathrm{L}$ DHB solution and $1 \mu \mathrm{L}$ of the mixture were spotted to the target plate for crystallization.

The mass spectrometry was performed using a Voyager DE Pro MALDI-TOF $\mathrm{N}_{2}$-Laser with a wavelength of $337 \mathrm{~nm}$ (Applied Biosystems, Foster City, USA). All spectra were measured in the linear detector mode. Laser intensities and the number of records per spectrum were varied manually.

Voyager software was used for data acquisition and peak detection. For quantification the peak intensity of each peptide was determined relative to the non-sialylated glycopeptide 1-32 (3459.66 Da) as internal standard. This peptide is detected in all spectra of the sialylation variants of the glycopeptides and is not changed by TS reaction (Figure 8). All measurements were repeated for at least three times.

\section{Homology Modeling}

A homology model of $T$. congolense TS1 e-1 was calculated using the software Yasara 10.11.8 [35-40]. The 
crystal structure of T. cruzi TS (UniProt: Q26964; PDB entry: 3B69), previously reported by Buschiazzo et al., 2002 [12] was used as the template structure. A benzoylated $\mathrm{N}$-acetylneuraminic acid derivative used as a ligand for $T$. cruzi TS in the template structure was kept in the binding site during the calculation of the homology model.

The following parameters of the Yasara homology modeling module were modified manually from the default settings of the program: Modeling speed: slow, PsiBLASTs: 6, EValue Max: 0.5, Templates total: 1, Templates SameSeq: 1, OligoState: 4, alignments: 10, LoopSamples: 50, TermExtension:10.

\section{Acknowledgements}

We like to thank Joel Smith, who initially searched TS genes in the T. congolense genome in the WTSI database. We gratefully acknowledge Prof. Patrick Ziegelmüller (University of Hamburg, Germany) for providing a clone of E. coli M15 (pREP4) expressing trans-sialidase from Trypanosoma cruzi. Neu5Ac-pNP were kindly donated by Prof. Joachim Thiem (University Hamburg, Germany). We are grateful to Evelin Tiralongo and Roland Schauer (University Kiel, Germany) for providing the T. congolense genomic DNA, Hans Lange and Hilmar Lemke (University Kiel, Germany) for the cell line producing anti-T. congolense TS antibody mAb 7/23. The plasmid vector pDEF was kindly provided by Prof. Paul Crocker (University of Dundee, UK). We would like to thank the German Federal Ministry for Education and Research (BMBF, project BioChangePLUS 031632A), the Tönjes-Vagt Foundation (project XXI) and Deutsche Forschungsgemeinschaft (DFG, project KE 428/8-1) for their financial support of this work.

\section{Authors' contributions}

HKB carried out all the HPAEC-PAD based TS assays and its data acquisition, the Siglec-4 binding assay and SDS-PAGE to determine resialylation of asialofetuin, homology modeling, alignment of amino acid sequences, supported MALDI data acquisition and drafted the manuscript. TTG carried out T. congolense TS1 cloning, sequencing and expression as well as T. cruzi TS expression. MW performed the TS1g enzyme assays. OR participated in development of the HPAEC-PAD based TS assay. PM carried out MALDI-MS analysis and its data acquisition. ED participated in MALDI-MS analysis and its data acquisition. FD established the pDEF-Transin-TS-3C-SNAP-Strep vector construct and carried out T. congolense TS1 cloning. SK designed and coordinated the study and supported drafting of the manuscript. All authors read and approved the final manuscript.

Received: 22 April 2011 Accepted: 30 July 2011 Published: 30 July 2011

\section{References}

1. Steverding D: The history of African trypanosomiasis. Parasit Vectors 2008, 1(1):3.

2. Schenkman S, Jiang MS, Hart GW, Nussenzweig V: A novel cell surface trans-sialidase of Trypanosoma cruzi generates a stage-specific epitope required for invasion of mammalian cells. Cell 1991, 65(7):1117-1125.

3. Montagna G, Cremona ML, Paris G, Amaya MF, Buschiazzo A, Alzari PM, Frasch AC: The trans-sialidase from the african trypanosome Trypanosoma brucei. Eur J Biochem 2002, 269(12):2941-2950.

4. Garcia ES, Azambuja P: Development and interactions of Trypanosoma cruzi within the insect vector. Parasitol Today 1991, 7(9):240-244.

5. Pontes de Carvalho LC, Tomlinson S, Vandekerckhove F, Bienen EJ, Clarkson AB, Jiang MS, Hart GW, Nussenzweig V: Characterization of a novel trans-sialidase of Trypanosoma brucei procyclic trypomastigotes and identification of procyclin as the main sialic acid acceptor. J Exp Med 1993, 177(2):465-474

6. Tiralongo E, Schrader S, Lange H, Lemke H, Tiralongo J, Schauer R: Two trans-sialidase forms with different sialic acid transfer and sialidase activities from Trypanosoma congolense. J Biol Chem 2003, 278(26):23301-23310
7. Engstler M, Schauer R: Sialidases from African trypanosomes. Parasitol Today 1993, 9(6):222-225

8. Nagamune $\mathrm{K}$, Acosta-Serrano A, Uemura H, Brun R, Kunz-Renggli C, Maeda Y, Ferguson MA, Kinoshita T: Surface sialic acids taken from the host allow trypanosome survival in tsetse fly vectors. J Exp Med 2004, 199(10):1445-1450.

9. Tiralongo E, Martensen I, Grotzinger J, Tiralongo J, Schauer R: Transsialidase-like sequences from Trypanosoma congolense conserve most of the critical active site residues found in other trans-sialidases. Biol Chem 2003, 384(8):1203-1213.

10. Eisenhaber B, Bork P, Yuan Y, Loffler G, Eisenhaber F: Automated annotation of GPI anchor sites: case study C. elegans. Trends Biochem Sci 2000, 25(7):340-341.

11. Cremona ML, Sanchez DO, Frasch AC, Campetella O: A single tyrosine differentiates active and inactive Trypanosoma cruzi trans-sialidases. Gene 1995, 160(1):123-128.

12. Buschiazzo A, Amaya MF, Cremona ML, Frasch AC, Alzari PM: The crystal structure and mode of action of trans-sialidase, a key enzyme in Trypanosoma cruzi pathogenesis. Mol Cell 2002, 10(4):757-768.

13. Green ED, Adelt G, Baenziger JU, Wilson S, Van Halbeek H: The asparaginelinked oligosaccharides on bovine fetuin. Structural analysis of $\mathrm{N}$ glycanase-released oligosaccharides by 500 -megahertz $1 \mathrm{H}$ NMR spectroscopy. J Biol Chem 1988, 263(34):18253-18268.

14. Frasch AC: Functional diversity in the trans-sialidase and mucin families in Trypanosoma cruzi. Parasitol Today 2000, 16(7):282-286.

15. Buschiazzo A, Campetella O, Frasch AC: Trypanosoma rangeli sialidase: cloning, expression and similarity to T. cruzi trans-sialidase. Glycobiology 1997, 7(8):1167-1173.

16. Engstler M, Schauer R, Brun R: Distribution of developmentally regulated trans-sialidases in the Kinetoplastida and characterization of a shed trans-sialidase activity from procyclic Trypanosoma congolense. Acta Trop 1995, 59(2):117-129.

17. Buschiazzo A, Tavares GA, Campetella O, Spinelli S, Cremona ML, Paris G, Amaya MF, Frasch AC, Alzari PM: Structural basis of sialyltransferase activity in trypanosomal sialidases. Embo J 2000, 19(1):16-24.

18. Amaya MF, Watts AG, Damager I, Wehenkel A, Nguyen T, Buschiazzo A, Paris G, Frasch AC, Withers SG, Alzari PM: Structural insights into the catalytic mechanism of Trypanosoma cruzi trans-sialidase. Structure 2004, 12(5):775-784

19. Damager I, Buchini S, Amaya MF, Buschiazzo A, Alzari P, Frasch AC, Watts A, Withers SG: Kinetic and mechanistic analysis of Trypanosoma cruzi transsialidase reveals a classical ping-pong mechanism with acid/base catalysis. Biochemistry-Us 2008, 47(11):3507-3512.

20. Brown WM, Saunders NR, Mollgard K, Dziegielewska KM: Fetuin-an old friend revisited. Bioessays 1992, 14(11):749-755.

21. Cointe D, Leroy Y, Chirat F: Determination of the sialylation level and of the ratio alpha- $(2->3) / a l p h a-(2->6)$ sialyl linkages of $\mathrm{N}$-glycans by methylation and GC/MS analysis. Carbohydr Res 1998, 311(1-2):51-59.

22. Dziegielewska KM, Brown WM, Casey SJ, Christie DL, Foreman RC, Hill RM, Saunders NR: The complete CDNA and amino acid sequence of bovine fetuin. Its homology with alpha $2 \mathrm{HS}$ glycoprotein and relation to other members of the cystatin superfamily. J Biol Chem 1990, 265(8):4354-4357.

23. Spiro RG: Studies on fetuin, a glycoprotein of fetal serum. I. Isolation, chemical composition, and physiochemical properties. J Biol Chem 1960, 235(10):2860-2869.

24. Zhang JQ, Biedermann B, Nitschke L, Crocker PR: The murine inhibitory receptor $\mathrm{mSiglec}-\mathrm{E}$ is expressed broadly on cells of the innate immune system whereas $\mathrm{mSiglec}-\mathrm{F}$ is restricted to eosinophils. Eur J Immunol 2004, 34(4):1175-1184.

25. Matrisian LM, Glaichenhaus N, Gesnel MC, Breathnach R: Epidermal growth factor and oncogenes induce transcription of the same cellular mRNA in rat fibroblasts. Embo J 1985, 4(6):1435-1440.

26. Walker PA, Leong LE, Ng PW, Tan SH, Waller S, Murphy D, Porter AG: Efficient and rapid affinity purification of proteins using recombinant fusion proteases. Biotechnology 1994, 12(6):601-605.

27. Thakar K, Niedenthal R, Okaz E, Franken S, Jakobs A, Gupta S, Kelm S, Dietz F: SUMOylation of the hepatoma-derived growth factor negatively influences its binding to chromatin. Febs J 2008, 275(7):1411-1426.

28. Kumar R, Stanley P: Transfection of a human gene that corrects the Lec1 glycosylation defect: evidence for transfer of the structural gene for $\mathrm{N}$ - 
acetylglucosaminyltransferase I. Molecular and cellular biology 1989, 9(12):5713-5717.

29. Neubacher B, Schmidt D, Ziegelmuller P, Thiem J: Preparation of sialylated oligosaccharides employing recombinant trans-sialidase from Trypanosoma cruzi. Org Biomol Chem 2005, 3(8):1551-1556.

30. Agusti R, Paris G, Ratier L, Frasch AC, de Lederkremer RM: Lactose derivatives are inhibitors of Trypanosoma cruzi trans-sialidase activity toward conventional substrates in vitro and in vivo. Glycobiology 2004, 14(7):659-670

31. Hirmo S, Kelm S, Schauer R, Nilsson B, Wadstrom T: Adhesion of Helicobacter pylori strains to alpha-2,3-linked sialic acids. Glycoconj $J$ 1996, 13(6):1005-1011.

32. Rocklin R, Clarke A, Weitzhandler M: Improved long-term reproducibility for pulsed amperometric detection of carbohydrates via a new quadruple-potential waveform. Anal Chem 1998, 70(8):1496-1501.

33. Bock N, Kelm S: Binding and inhibition assays for Siglecs. Methods Mol Biol 2006, 347:359-375.

34. Laemmli UK: Cleavage of structural proteins during the assembly of the head of bacteriophage T4. Nature 1970, 227(5259):680-685.

35. Canutescu AA, Shelenkov AA, Dunbrack RL Jr: A graph-theory algorithm for rapid protein side-chain prediction. Protein Sci 2003, 12(9):2001-2014

36. Jones DT: Protein secondary structure prediction based on positionspecific scoring matrices. J Mol Biol 1999, 292(2):195-202.

37. King RD, Sternberg MJ: Identification and application of the concepts important for accurate and reliable protein secondary structure prediction. Protein Sci 1996, 5(11):2298-2310

38. Krieger E, Joo K, Lee J, Raman S, Thompson J, Tyka M, Baker D, Karplus K. Improving physical realism, stereochemistry, and side-chain accuracy in homology modeling: Four approaches that performed well in CASP8. Proteins 2009, 77(Suppl 9):114-122.

39. Muckstein U, Hofacker IL, Stadler PF: Stochastic pairwise alignments. Bioinformatics 2002, 18(Suppl 2):S153-160.

40. Qiu J, Elber R: SSALN: an alignment algorithm using structure-dependent substitution matrices and gap penalties learned from structurally aligned protein pairs. Proteins 2006, 62(4):881-891.

doi:10.1186/1471-2091-12-39

Cite this article as: Koliwer-Brandl et al:: Biochemical characterization of trans-sialidase TS1 variants from Trypanosoma congolense. BMC

Biochemistry 2011 12:39.

\section{Submit your next manuscript to BioMed Central and take full advantage of:}

- Convenient online submission

- Thorough peer review

- No space constraints or color figure charges

- Immediate publication on acceptance

- Inclusion in PubMed, CAS, Scopus and Google Scholar

- Research which is freely available for redistribution

Submit your manuscript at www.biomedcentral.com/submit 